\section{Bei der spezifischen Immuntherapie Verwandtschaft ausnutzen!}

V on Mitte Mai bis Anfang August fliegen in Deutschland Gräserpollen. Dabei handelt es sich überwiegend um Arten aus der Unterfamilie der Pooideae, die phylogenetisch eng verwandt sind. Für die Hyposensibilisierung bei Gräserpollenallergie werden in Europa überwiegend Gräsermischungen eingesetzt, die das Spektrum der wichtigsten allergierelevanten Gräserarten abdecken. Klinisch bewährt hat sich die Fünf-Gräser-Mischung aus Dactylis glomerata (Wiesenknäuelgras), Anthoxanthum odoratum (Gewöhnliches Ruchgras), Lolium perenne (Deutsches Weidelgras), Phleum pratense (Wiesenlieschgras) und Poa pratensis (Wiesenrispengras). Diese Mischung ist auch im Sublingualpräparat Staloral ${ }^{\circledR} 300$ sowie in der Allergentablette Oralair ${ }^{\circledR}$ Gräser enthalten. Die klinische Wirksamkeit und Sicherheit dieser Allergenpräparaten ist gut dokumentiert.

In Deutschland wird häufig die spezifische Immuntherapie von Patienten mit einer Roggenpollenallergie noch mit

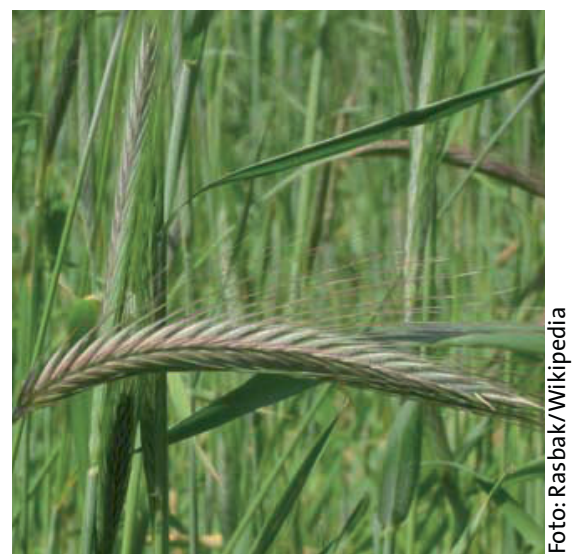

Roggen (Secale cereale) gehört wie die allergologisch relevanten Gräserarten zur Familie der Pooideae.

speziellen Roggenpollenextrakten durchgeführt - oft zusätzlich zu einer Hyposensibilisierung mit einer Gräserpollenmischung. Roggen (Secale cereale) gehört ebenfalls zur Unterfamilie der Pooideae. Wie die übrigen Pooideae-Spezies enthalten auch Roggenpollen Majorallergene sowohl aus der Allergengruppe 1
(Sec c 1) als auch der Gruppe 5 (Sec c 5). In Immunoblot- und ELISA-Untersuchungen mit den gepoolten Seren von 15 Patienten mit Gräserallergie konnte eine vergleichbare Hemmung der spezifischen IgE-Bindung von Roggenallergenen durch die Fünf-Gräser-Mischung wie auch durch einen Roggenpollenextrakt erreicht werden. Diese Befunde sprechen für eine hohe Kreuzreaktivität von Gräser- und Roggenallergenen. Die hohe Übereinstimmung der IgE-Reaktivität von Allergenen aus Secale cereale und den Allergenen der Fünf-Gräser-Mischung wird weiter gestützt durch Untersuchungen mit murinen monoklonalen Antikörpern, bei denen sich ebenfalls eine hohe Kreuzreaktivität zwischen den Allergenen zeigte.

Die hohe Kreuzreaktivität zwischen Roggen und Gräsern kann man sich therapeutisch zunutze machen: Patienten mit einer Roggenpollenallergie profitieren auch von einer Hyposensibilisierung mit der Fünf-Gräser-Mischung. Ein solches Vorgehen hat auch gesundheitsökonomische Vorteile.

Nach Informationen von Stallergenes, Kamp-Lintfort

\title{
Wirkung bis in die kleinsten Atemwege
}

\begin{abstract}
Mision it dem Asthmapräparat Inuvair ${ }^{\circledR}$ Mikrosol stehen das Glukokortikoid Beclometason und der lang wirksame $\beta_{2}$-Agonist Formoterol als Fixkombination zur Verfügung. Damit erreichen Asthmapatienten bereits unter vergleichsweise geringer Glukokortikoiddosierung die angestrebte Symptomkontrolle, berichtete Dr. Thomas Voshaar, Moers. Da die Wirkstoffe vollständig gelöst sind, können sie feiner vernebelt werden als bei herkömmlichen Suspensionsaerosolen. So werden besonders feine Partikel mit einer Größe von nur 1 bis $2 \mu \mathrm{m}$ erzeugt, die bis tief in die kleinsten Atemwege vordringen kön-
\end{abstract} nen.

In einer randomisierten klinischen Studie hat Inuvair ${ }^{\circledR}$ mit seiner guten Lungendeposition im direkten Vergleich mit der freien Kombination der beiden Wirkstoffe Beclometason und
Formoterol eine mindestens ebenbürtige Wirksamkeit unter Beweis gestellt. Patienten, die die Fixkombination erhalten hatten, berichteten sogar über signifikant mehr symptomfreie Tage und signifikant mehr Tage mit kompletter Asthmakontrolle als diejenigen Patienten, die die Wirkstoffe separat inhaliert hatten.

Fixkombinationen aus Glukokortikoid und lang wirksamen $\beta_{2}$-Agonisten haben auch für die Patienten einen hohen Stellenwert, das bestätigt eine aktuelle Umfrage unter 1.000 Asthmakranken. Wie Prof. Dr. J. Christian Virchow, Rostock, berichtete, gaben 91\% der Befragten an, dass eine Fixkombination die Therapie im Vergleich zur getrennten Inhalation vereinfache. Wegen der praktischen Handhabung und der guten Wirkstoffverteilung in der gesamten Lunge bevorzugen die
Fixkombination im Doppelpack Die Fixkombination Inuvair ${ }^{\circledR}$ ist seit Oktober 2008 auch als Doppelpackung erhältlich. Dies ermöglicht mehr Flexibilität in der Verordnungsdauer: Der Arzt muss das Medikament nicht jeden Monat neu verschreiben. Dennoch behält er die Asthmakontrolle seines Patienten im Blick, da dieser alle zwei Monate ein Wiederholungsrezept benötigt und in der Praxis vorspricht. Ein Bonus für den Patienten ist die geringere Zuzahlung beim Doppelpack.

Betroffenen Lösungsaerosole. Zwei Drittel der befragten Patienten würden lieber ein Aerosol als ein Pulver inhalieren.

aad

2. Janssen-Cilag Asthma-Colleg „AsthmaTherapie, die beim Patienten ankommt". Frankfurt/Main, 27. August 2008.

Veranstalter: Janssen-Cilag, Neuss 